\title{
Applied Algorithmization in Optimization of Airport Processes Simulation Models
}

\section{František Adamčík ${ }^{1}$, Jozef Galanda ${ }^{2}$, Radoslav Šulej2*, Martin Jezný $^{3}$}

\author{
${ }^{1}$ Department of Avionics, Faculty of Aeronautics, Technical University of Košice, Košice, Slovak Republic \\ ${ }^{2}$ Department of Air Transport Management, Faculty of Aeronautics, Technical University of Košice, Košice, \\ Slovak Republic \\ ${ }^{3}$ Airport Košice, a.s., Košice, Slovak Republic \\ *Corresponding author: Technical University of Košice, Faculty of Aeronautics, Department of Air Transport \\ Management, Rampova 7, 04121 Košice, Slovak Republic, Email: radoslav.sulej@tuke.sk
}

\begin{abstract}
The authors of this contribution are dealing with the potential of applied algorithmization as a methodology of improving efficiency and accelerating the process of designing as well as program implementation of simulation models for the purpose of computer-based modeling and simulation of airport processes. At this simulation, quality is built upon on a precisely defined model of airport processes are set forth by the authors as part of the process of designing a modern Departure Control System (DCS) of airports, which currently designated for automation of a complex management of processes in the field of passenger and baggage handling, the airports offer to individual air carriers. The resulting algorithms designed for the separate separate processes of the DCS will help the authors understand real processes taking place at the airport with the aim to optimize the course of actions at the airport operation. A precisely built model of airport processes is the prerequisite of proper implementation of the simulation, pointing out both the advantages and disadvantages of this models and their implementation in practice.
\end{abstract}

Keywords

Airport processes - Algorithmization - Departure Control System - Simulation — Simulation model

\section{Introduction}

Maintaining competitiveness and improving the level of services provided is the requirement that is calling for continuous changes to be made by the organization. Under the conditions of strict cost-monitoring, i tis necessary to verify the potentials of planning systems and find innovative and successful solutions. Requirement for changes in technology or organizational processes, however, some risks are involved. Limiting i tis possible by way of predictive technologies and simulation meth- ods that enable modeling of the work environment a simulation of the outcomes of various decisions. All that results in a higher level of trust in the suggested solution is fitting for the organization before the implementation oft it has been launched. The role of simulation is to evaluate the real options available either in support of strategic stimuli that could involve incurring a large amount of finances or those promoting continuous search for better performance at operational and tactical levels. Simulation provides its user a wider breadth and depth 
of information on which decision is based. Development of a simulation model is a creative and intuitive process, where your abilities and skills can be fully implemented. However, developing a simulation model is only the basic step. Simulation is primarily a process of experimenting with the model for the purpose of either understanding the behavior of the system of evaluating the various strategies of the system operation.

\section{Airport Processes}

Airport processes involve activities taking place at airfields and related to handling or supporting processes. The notion of airport processes can also be understood as a series of steps or events the last for a certain period of time also occupying some areas of the airport and are subjected to some survey bringing some results. The process can be identified on the basis of changes made in the properties of one or several objects influenced. Handling process is defined as provision of handling services by the air carrier. These processes and their item are detailed in the Airport Handling Manual (AHM), stating what in this process is involved, in what sequence and what kind of documentation is to be filled in, and wt kind of reports are to be transmitted, and who is taking the responsibility for the various tasks [1], see figure 1. The airport handling process can be divided into:

- Business handling: passenger, baggage and cargo handling

- Technical handling: guidance of the aircraft to the stand, refueling of the aircraft, connecting to the auxiliary ground power unit (GPU), addition of the stair-step, deicing, anti-icing procedures etc.

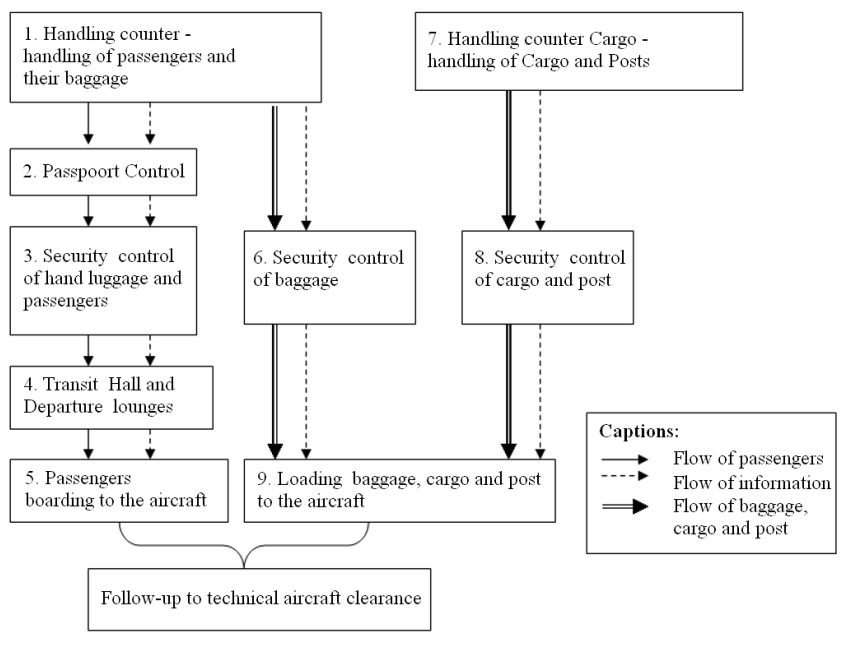

Figure 1. Block diagram of the traffic handling [2]

Airport operation, however, does not include activities of the airport operator, who is responsible fo the coordination of all the subjects present at the a the airport (as stipulated by $\S 32$ of the Air Law of the SR, but also other organizations participating in the operation as well as those also participating in the operation and doing their job in the controlled area and mainly in the air side of the airport. In this case, the notion of "airport operation" involves processes related to the air traffic operation and services. The level of security protection and quality of services depend on, the system of airport precesses adopted and how the individual subjects are capable of communicating and communicating with one another as a result.

The notion of the system in this case is understood as stated and established rules for performing the individual airport processes. However, it does not necessarily only the sole responsibility of the airport operator to "develop" the entire system of airport processes alone. Its role is to create a framework of rules and manage the harmonization of procedures and activities run at the airport, whereas the main priority should remain with the air traffic safety. Cooperation of these subjects is inevitable particularly during operationally demanding activities such as poor visibility, handling dangerous cargo and emergency (distress) situations [3].

When analyzing the airport processes, we are concentrating research to find answers mostly to questions such as the parts of the airport process is made up, the input parameters, and the parameters of the individual segments of the airport processes, measuring them and how these can be affected by the conditions and what variables and constants are encountered during the operations, what result is sought after, and in what units of measurement the measured result is, and to what extend the foun result can be used for further task such as: managerial (tasks of direction), optimization (emphasis placed on efficiency), quantitative(amount-capacity), qualitative (achieving qualitative indicators), safety (ensuring safety in the fields of safety and security).

What airport processes are necessary to be analyzed? They are airport processes directly involved with providing services for the airport customers (travelers and air carriers), involving particularly handling of passengers and baggage, cargo and post as well as services of ground handling and airport processes directly linked with ensuring airport operation, in particular those related to collection, submission and publication of statistical data, access to the air side of the terminal and the airport moving areas, ground operation on the ground, rules governing movements of persons and vehicles on the airport operational areas, security management in the handling area, operation at low visibility, biological protection of the airport, manipulation with dangerous materials, checking movement areas and visual navigational aids, maintenance of movement and operationally important areas of the airport, maintenance of visual navigation aids and devices, winter operation of the airport, emer- 
gency plan of the airport, first-aid and fire protection services and removal of aircraft unable of movement.

\section{Applied Algorithmization of Airport Processes}

The information system is a means for automated solving of tasks based on information involving processes at airports in civil aviation as well. The process of performing operations is named as the account of the airport processes. The solution of the task is often formulated so that the the sequence of the tasks remains not quite clear. Therefore, it is necessary to determine the exact rules applicable to the sequence of the partial operations. This process is called algorithmization of the tasks of airport processes. In order to solve the individual airport processes, we are trying to find an algorithm, i.e. to find a unified sequence of actions, which are to be performed so as to find, at the end of a finite number of steps, the solution sought after to the tasks related to the airport processes. We are also aware of the problems that can or cannot be solved algorithmically. We are interested only in the solvable ones, those that can be solved on computers in real time. Solving a tas of airport processes means transforming the input data into output ones following a sequence of steps i.e. in a single moment only a single elementary step is performed. Both the input and output data are defined by input and poutput characteristics conditions.

The algorithm of the airport processes is affected by various parameters. Some of them must be defined and clearly measurable, so that they can help defining the system. The parameters themselves can directly afect the entire process (the so-called influential conditions). The algorithm of the airport precesses as a whole must be capable of producing an output (the so-called result sought after). It must be determined so that to be comprehensible as to what we are to arrive at and it is important to determine the goal of the problem solved [3].

For example, if the object of the solution is the airport process related to passenger handling, the parameter of an optimal algorithm can also be e.g. a number of the handling counters, attendants of the check-in, capacity of the handling counters, or that of the passengers security and detection control, number of the check-in staff or departure gates etc. For example, an influential condition can also be the number of travelers, capacity of the check-in counter, security and detection control, time of handling passengers for the appropriate flight or the requirement of the air carrier to start the process of handling. And as an output, the results sought after can be answers to the questions like what is the overall capacity of check-in counter? How much time does it take to handle passengers for a given flight? Or what is the required number of check-in counters or staff and what were the possibilities of optimizations in practice? On the other side, the object of search are solutions of the airport processes in the areas of handling baggages, while parameters of the optimal algorithm can be numbers such as the number of check-in counters, baggage trolleys, number of the staff in the sorting or screening room, the average number of an weight of baggages per passenger, capacity of the handling counter, that of the security and detection control of baggages, capacity of baggage trolleys, requirements of air carriers over starting the handling process etc. The results sought after can be answers to the questions: What is the necessary capacity for the baggage sorting room? How much time does it take to handle baggage for a give flight? What is the necessary number of baggage trolleys? What is the necessary number of for the baggage sorting room? What are the potentials of applying optimization in practice? The most important in terms of the quality of the algorithm is an exact and clear defining of the individual characteristics of the airport processes [3].

\section{Applied Algorithnmization and Departure Control System}

Departure Control Systems (DCS) are currently designed for automated and complex management of the airport passenger and baggage handling. Flawless handling makes it inevitable to cooperate with the passengers as their approach to the process of handling can either expedite for slow down to it. Handling services are provided by airports or air carriers on an individual basis. The ambitions of the problem solvers, based on the research of the offered services and the levels of introducing the Local Departure Control System in practice and the contacts with their users and requirements is to obtain information for designing a new, competitive solutions of DCS of own design, that which thanks to its features and supports provided by the most modern and verified technologies would meet the highest criteria of reliability and safety in the areas of their employment [4].

In the process of project solution, we are engaged in designing the possible variants of solutions of algorithms of the individual modules of the DCS and its software under development. Our primary goal is is to make maximum use of open standards, either of SW or HW and following a development process we are assembling the resulting algorithms into their final and feasible form suitable for fast and efficient realization of the resulting code of solving algorithms for the modules of the DCS. The design of the basic system concepts illustrated in figure 2. Thanks to the suitable use of the methods of applied algorithmizationm, and appropriate observance of all the rules of setting up all the algorithms and maintaining all the features of algorithm, the research team has managed to find a phase sequencing of the sepa- 
rate airport processes into elementary activities, which could be program processed. By logical arrangement of the interlinked activities into a logical sequences, we have managed to develop the individual algorithm of the airport processes with the aim to achieve efficient processing into the program code within the individual modules of implementing the airport processes. Thanks to the optimal complexity and resulting high efficiency of developed independent algorithms, it is possible to apply not only the implementation of individual program modules of the resulting DCS, but also as a basis for potential simulation models. By way of suitably applied methodology, this simulation models can be subsequently used for thinning out and realization of airport processes for the need of further research and needs of the practice. Quality of such simulation is then strictly dependent on the quality of the designed and subsequently implemented simulation model. As the demonstration of the applied methodology of algorithmization, we are providing the possible solution pattern pf one of the most frequent airport processes, namely the process of passenger handling at the check-in with the requirement to start the handling process 90 minutes before the aircraft departure.

\subsection{The issue raised}

An air carrier $\mathrm{X}$ providing chartered flight from airport "A" to airport "B" is departing with 70 passengers. As by the Handling contract concluded with the airport "A" there are two check-in counters for the passengers and the requirement to start the handling process 120 prior to the departure of the aircraft under the condition that the check-in counter No. 2 will be open only after the elapsing of the first hour of the handling process on condition that not all of the passengers have been handled during this first hour of handling at counter No. 1. Capacity of the airport Check-in Counter 1 is a passenger per 2 minutes. Cyclical interval is 10 minutes.

The result sought after - answers to the following questions:

1. How many passengers (PAX) can be handled 60 minutes after starting the handling process at checkin counter No. 1 ?

2. How many passengers (PAX) will remain unchecked after 60 minutes of starting the handling process at check-in counter No. 1 ?

3. What is the total time of handling all the passengers?

4. What is the lead-time for all the passengers to be handled in time?

\subsection{Algorithmization of the Process}

The solution of the problem and it subsequent algorithmization, which can serve as a simulation model in the deployment phase and a subsequent simulation can help finding requires answers presented in figure 2 .

In presented figure 2, $\mathrm{A}$ is the number of passengers not handled; $\mathrm{C} 1$ is total time of the handling process; $\mathrm{C} 2$ is lead-time; D is time of starting the handling process before flight; CI is cyclical interval; $\mathrm{Pr}$ is capacity of the check-in counter; PrCI is capacity of the check-in counter for the unit of cyclic interval and Num is number of the passengers handled.

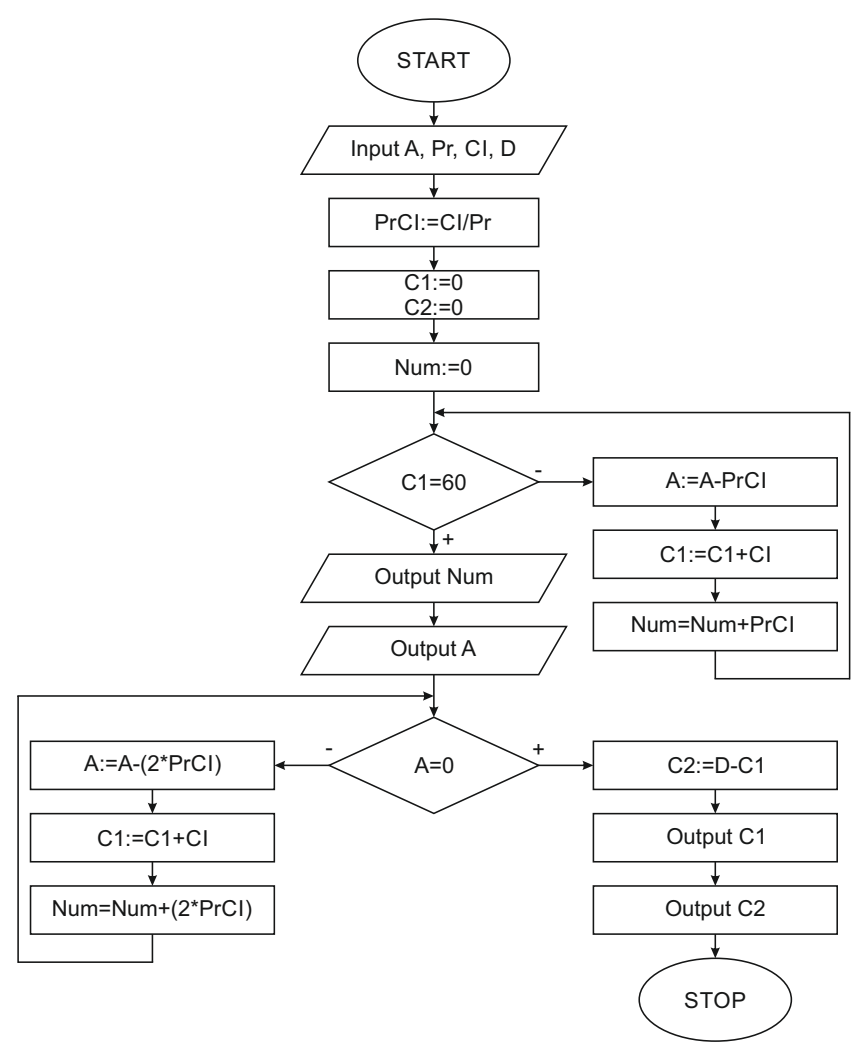

Figure 2. Algorithmization of the process

\subsection{Description of algorithmization of the process and} the functioning of the simulation model

Having downloaded the input variables the PrCI variable is entered the results of the devision of the cyclical interval and the capacity of the handling counter. Inserted into the total time of the handling is 0 , into the lead time again 0 and equally 0 into the number of handled passengers. The follows a cycle, which fill be repeated until the total time of the handling process will equal to the value of 60 . If it equals to 60 , written on the screen the number of the passenger handled will be seen as well those not handled. Then follows another cycle, investigating if the the number of un-handled passengers is equal to 0 . If not, several computations are made. If not, the variable inserted into variable $\mathrm{C} 2$ is the value from the count-down of the time from the beginning of the handling process with respect to the total time of handling prior to the departure. The screen will show 


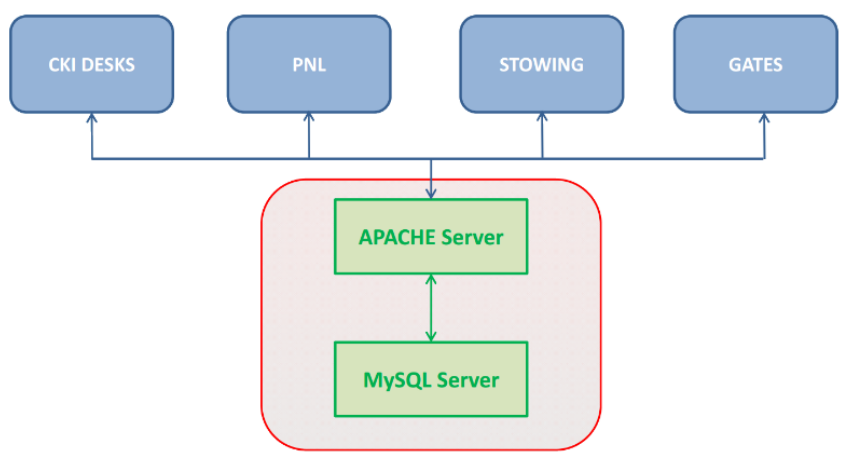

Figure 3. Main WS core topology

the total time of the handling process and the lead-time. Subsequently, the algorithm is ended.

Similar way had to be applied, using the same methodology, to other airport processes, which were to be taken into account when designing the individual functions of the DCS under development. The level and precision of analyzing the individual processes are inevitable to form a basis of subsequent phasing of the processes into partial activities, which can be simply algorithmized. Their synthesis is the precondition to a high level code and the resulting quality of the simulation model of the aviation process.

Currently, the research team is working on the most suitable and optimal specification of the HW oriented on an open operation system Linux both for the server platform, and the CKI, gate and WAB terminals. The design of the basic concept for the system is shown in figure 3.

In the same way, we are inevitalble taking into account the optimal dimensioning of hardware componsnts of the server and workstations so as to maintain all the technical and seceurity requirements for conservation of functianality of the overall resulting DCS as a system.

\section{Conclusion}

Scientific methods are tools of any scientific trade. Without them, it is impossible to obtain exact, mutually interwoven facts, termed as the basic requirements established for science. In the process of developing the structure of the model of airport processes, almost all the the generally applicably scientific methods have been used used. Of equal importance is the perfect knowledge of the investigated systems, primarily the mutual links among the entities, stock variables and the system components with one another. A precisely built model of the airport processes with a correctly defined structure is the fundamental prerequisite to a correctly realized simulation.

Based on the analyses of the requirements of potential customers of the system and removing those of the inefficient or those that cannot be materialized, we have built several possible variants of solutions and developed the individual algorithm of the required airport processes. Only when comparing the variants the problematic algorithms could have been revealed, that we have subsequently corrected. Thanks to the techniques of suitable algorithmization of the individual processes and their decomposition into the separate activities, it was possible to simplify the given process and accelerate it for the most part. These have been subjected to reviews by potential clients. Based on a subsequent discussion, it was possible to choose the optimal. On checking for feasibility, the chosen variant has been developed in detail with the subsequent specification of the components and software modules needed to accomplish the project goals. The resulting algorithms of the individual processes the DCS as suggested enable the authors to find better understanding for the real airport processes with the aim to optimize the course of actions in the airport operations.

\section{Acknowledgments}

This work was supported by the Slovak Research and Development Agency under the contract No. APVV-150527 - "New generation of departure control system for airports". The authors would like to thank the project management and colleagues for support and valuable questions, remarks and suggestions.

\section{References}

[1] J. Galanda, R. Šulej, and M. Jezný. 3D computer model of košice airport and its usage in simulations of airport processes. In Proc. of 11th international scientific conference New Trends in Aviation Development, pages 1-6, Košice, Slovakia, 2014. Technical University of Košice. ISBN 978-80-553-1650-5.

[2] L. Kerner. Provozní aspekty letišť [Operational aspects of airports]. CTU Publishing House, Prague, 2003. ISBN 80-01-02841-0. [In Czech].

[3] J. Galanda, R. Šulej, and M. Jezný. Airport operation staff training utilizing simulations of the defined airport processes. In Proc. of 10th International Multi-Conference on Society, Cybernetics and Informatics (IMSCI 2016), pages 290-296, 2016. ISBN 978-1-941763-44-5.

[4] F. Adamčík et al. New generation of the departure control system for airports. In Proc. of 13 th international scientific conference New Trends in Aviation Development, pages 1-5, Košice, Slovakia, 2016. Technical University of Košice. ISBN 978-80-553-2628-3. 